\title{
Elements of a competition
}

\author{
To join in with the International Year of Chemistry celebrations, we launched a competition earlier this \\ year inviting aspiring science communicators to write an essay about one of seven elements.
}

Each month, the last page of Nature Chemistry is occupied by an article called 'In Your Element', which focuses on a chemical element. These pieces typically contain interesting snippets about the element's discovery, its reactivity, ongoing research related to it or some of its applications. Of course, each article should be scientifically and historically sound, but rather than presenting a humdrum list of dates and facts, or a technical report of an element's characteristics and uses, these essays are meant to be entertaining light reads.

With the backdrop of the International Year of Chemistry ${ }^{1}$ and our desire to see more scientists engage in outreach activities and communication ${ }^{2}$, we hoped that an essay competition $^{3}$ would coax some aspiring writers to try their hand at producing 700800 words about an element. Our eligibility criterion was that it should be no more than five years since the end of the entrant's final training appointment, reflecting the fact that we hoped to encourage younger chemists to get involved in communicating science.

The Nature Chemistry editorial team settled on seven elements for the competition: helium, nitrogen, sodium, copper, bromine, indium and plutonium. This list, which offered a wide range of characteristics for the entrants to discuss, was drawn from across the breadth and depth of the periodic kingdom - gases, solids and a liquid at room temperature; an alkali metal, a transition metal, and non-metals; highly reactive, non-reactive and radioactive.

By August 1, the deadline for submissions, we were delighted to see that many young scientists had accepted the challenge. We received almost one hundred essays, nearly half of which came from students currently pursuing $\mathrm{PhD}$ or Masters degrees, a quarter from undergraduate students, and even two from enterprising high-school students. Collectively, these contributions were from writers with very different backgrounds - from atmospheric to pharmaceutical chemistry (for those who indicated a speciality) - and from no less than twenty-five different countries, the top five being the USA, the UK, India, Germany and Canada (considering the writers' affiliations rather than their nationality).

All seven elements were well represented, but copper turned out to be the most popular element ( $26 \%$ of the entries), with nitrogen a close second (21\%). Turning to the cover letters for clues as to why - we had asked entrants to tell us a little about their reasons for picking a particular element - it seems that many writers chose an element that was somehow related to their own research. And copper and nitrogen are undoubtedly encountered more frequently in typical research projects than, say, plutonium.

\section{We thank all those who participated and encourage everyone to keep writing!}

A desire to highlight exciting aspects of very familiar elements also seemed to play a role. Copper has been known to man for thousands of years, and nitrogen not only makes up about $78 \%$ of the Earth's atmosphere but is also crucial in modern agriculture. Although their ubiquity might make them seem a little more ordinary than their periodic brethren, both elements are still exciting in their own right. To take but one example for each, copper plays an important part in superconducting materials, and the fixation and reduction of dinitrogen continues to challenge chemists.

A similar intention to do justice to seemingly common-or-garden elements was often mentioned by those who chose sodium ( $8 \%$ of the entries) as their subject. Although it is found in almost every laboratory, and even in any kitchen, its chemistry is far from dull. Sodium provided these writers with many anecdotes - from a drastic change in its properties under high pressure to its regulatory role in biological systems - to make for engaging essays.

Helium and indium each represented 13\% of the total number of submissions. It seems that children amazed at gravity-defying balloons, and amused at high-pitched voices, have grown up to remain intrigued by helium's physical properties - which become really bizarre below about $2 \mathrm{~K}-$ and impressed by its crucial role in quantum chemistry. Leaving helium to the physical chemists, materials scientists along with fervent supporters of flat-screen and touchscreen technologies turned more often to indium. In addition to its increasing role in new 'information age' devices, its emission of a 'crying' sound when bent also captured the attention of some of the writers.

Many chemists are drawn to the duality of elements - this aspect has come up in previous 'In Your Element' articles. Take phosphorus ${ }^{4}$, for example, whose elemental form $\mathrm{P}_{4}$ and organophosphate esters (known as nerve agents) have been put to terrible use as weapons of war, yet in phosphates serve as essential nutrients for living organisms. Similarly, bromine - named after its particularly unpleasant smell - is extremely useful in synthetic chemistry, but so toxic that its use has to be regulated to protect the environment. Bromine attracted $11 \%$ of the entrants, whereas $8 \%$ endeavoured to highlight the more positive aspects of another notoriously destructive element, plutonium. Only discovered about 70 years ago, it also offers much scope for the investigation of its properties, which are unusual even for a transuranic element.

Most writers also made a real effort with titles - quite a few seem to share our fondness for alliterations or puns - and suggested images to accompany their essays. Taken together, all of this made for a process of selecting winning entries that was far from easy. All of the editors of Nature Chemistry participated, as well as two external judges, Michelle Francl and Matthew Hartings, previously introduced on our blog ${ }^{5}$. The Nature Chemistry editorial team first whittled the list down by selecting the best three essays for each element, which were then ranked by four judges (two editors, Francl and Hartings). We are delighted to have one winning essay per element, and we now intend to publish them over the next year as part of the regular 'In Your Element' section of the journal, starting with sodium on the last page of this issue.

We congratulate the writers whose essays were selected to be published in the journal, and thank all those who participated and made this an exciting competition. And we encourage everyone to keep writing!

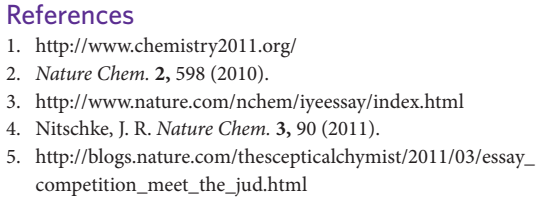

